\title{
Combinatorial Dyson-Schwinger equations in noncommutative field theory
}

\author{
Adrian Tanasă and Dirk Kreimer
}

\begin{abstract}
We introduce here the Hopf algebra structure describing the noncommutative renormalization of a recently introduced translation-invariant model on Moyal space. We define Hochschild one-cocyles $B_{+}^{\nu}$ which allows us to write down the combinatorial DysonSchwinger equations for noncommutative quantum field theory. One- and two-loops examples are explicitly worked out.
\end{abstract}

Mathematics Subject Classification (2010). 05E15, 81T18.

Keywords. Noncommutative quantum field theory, combinatorial Dyson-Schwinger equation, Moyal space, renormalization, Hopf algebras, pre-Lie and Lie algebras.

\section{Introduction}

Hochschild cohomology was shown, in the context of commutative quantum field theory (QFT), to play an important rôle in the understanding of different perturbative and non-perturbative issues [3], [23]. Using suitable Hochschild 1-cochains $B_{+}^{\gamma}$, one can thus write down the combinatorial Dyson-Schwinger equation, extending perturbative to non-perturbative physics.

On the other hand, noncommutative geometry is an interesting framework for both mathematics and theoretical physics (see for example [7], [9]). Noncommutative quantum field theory (NCQFT) on Moyal space has recently gained attention through the proposition of several models which were proved to be perturbatively renormalizable. Thus, despite the ultraviolet/infrared mixing problem [26] (a new type of non-local divergence which appears when implementing QFT on the Moyal space), several models are now known to be renormalizable. A first such model is the Grosse-Wulkenhaar model [13], which however explicitly breaks the translationinvariance of QFT. Recently, a translation-invariant model was proposed in [14]; this new model was also proved to be renormalizable at any order in perturbation theory [14].

The Hopf algebra structure of perturbative renormalization was implemented for the Grosse-Wulkenhaar model in [33]. In this paper we first repeat this for the 
translation-invariant model [14]. This task is more involved because of a more complicated power counting mechanism. We then go further and introduce Hochschild 1-cocyles $B_{+}^{\gamma}$ adapted for this noncommutative framework. This allows us to write down the combinatorial Dyson-Schwinger equations for both these types of noncommutative models. Nothing here involves mathematical sophistication beyond what was necessary for commutative field theory. But finer technical details have to be clarified, and are done so here by explicit example.

This paper is structured as follows. The next section recalls the definition of the Moyal space and lists the field theoretical models known so far to be renormalizable in this noncommutative frame. Particularly, we recall the translation-invariant model introduced in [14]. In Section 3 we introduce the Hopf algebra structure which describes the renormalization of this noncommutative model. The pre-Lie and Lie algebra structures associated to graphs are also presented. Section 4 analyzes in detail several differences (from a diagrammatic point of view) which appear when one uses the ribbon graph representation of NCQFT instead of the usual Feynman graphs of commutative QFT. In Section 5 we introduce Hochschild 1-cocyles $B_{+}^{\gamma}$ which allow to write down the combinatorial Dyson-Schwinger equations in NCQFT. We give here the corresponding theorems; note that these results hold for all renormalizable noncommutative models listed in Section 2. Finally, in the last section we completely work out as the crucial part of this paper the one- and two-loop implementations of the theorems of Section 5.

\section{Scalar field theory on the Moyal space and renormalizability}

In this section we briefly recall the definition of the Moyal space; we then list the field theoretical models (translation-invariant or not) known so far to be renormalizable on it.

The noncommutative Moyal space is given by

$$
\left[x^{\mu}, x^{\nu}\right]_{\star}={ }_{l} \Theta^{\mu \nu},
$$

where the noncommutative matrix $\Theta$ writes

$$
\Theta=\left(\begin{array}{cccc}
0 & \theta & 0 & 0 \\
-\theta & 0 & 0 & 0 \\
0 & 0 & 0 & \theta \\
0 & 0 & -\theta & 0
\end{array}\right) .
$$

Note that by $\star$ we denote the Moyal-Weyl product.

\subsection{The "naive" $\phi^{\star 4}$ model; Feynman graphs (planarity and non-planarity)}

- ribbon graphs. In order to obtain field theory on this space, the first thing that 
comes to mind is to replace the ordinary commutative local product of fields by the Moyal-Weyl product

$$
S[\phi]=\int d^{4} x\left(\frac{1}{2} \partial_{\mu} \phi \star \partial^{\mu} \phi+\frac{1}{2} \mu^{2} \phi \star \phi+\frac{\lambda}{4 !} \phi \star \phi \star \phi \star \phi\right) .
$$

Note that an Euclidean metric is used.

In momentum space the action (2.1) writes

$$
S[\phi]=\int d^{4} p\left(\frac{1}{2} p_{\mu} \phi p^{\mu} \phi+\frac{1}{2} \mu^{2} \phi \phi+\frac{\lambda}{4 !} \phi \star \phi \star \phi \star \phi\right) .
$$

An important consequence of the utilization of the non-local product $\star$ is that the interaction part does not longer preserve the invariance under permutation of incoming (outgoing) fields. This invariance is restricted to a cyclical permutation.

Furthermore, there exits a basis - the matrix base - of the Moyal algebra for which the Moyal-Weyl product becomes an ordinary matrix product. For these reasons, an appropriate way to draw the associated Feynman graphs is to use ribbon graphs, that is to use ribbons instead of lines for the propagators.

Thus, the "usual" commutative $\phi^{4}$ vertex becomes some ribbon $\phi^{\star 4}$ vertex as shown in Fig. 1.

Figure 1. The local vertex is replaced in NCQFT with a non-local, Moyal vertex.

This has important consequences on the definition of the pre-Lie structure of insertions of such ribbon graphs (see Section 3.2). Let us first give an important topological definition of these ribbon graphs:

Definition 2.1. A planar graph is called regular if it has a single face (the external one) broken by external edges.

While this definition takes recourse to the parlance in noncommutative field theory, it just takes into account standard combinatorial facts: Let us call a graph a genus- $n$ graph if it can be drawn without self-intersection on all surfaces with genus $\geq n$. Then a planar graph is genus- 0 .

Now, a genus- $n$ graph can be drawn on a surface of genus $n$ without self-intersections. Its internal edges and vertices hence allow for a unique labeling of faces on this genus $n$ surface. Let $k$ be the number of such faces which contain an external edge of the graph. A planar regular graph is a genus-0 graph with $k=1$. If $k>1$, we call the graph irregular. An example of a 2-point planar irregular graph is the tadpole of Fig. 2. 


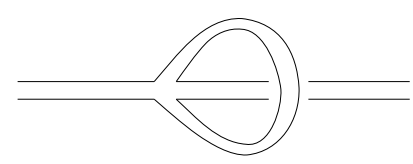

Figure 2. The "non-planar" tadpole. This graph is planar irregular (it has two broken faces).

2.2. The Grosse-Wulkenhaar-like models. The Grosse-Wulkenhaar model is a scalar quantum field theory on the four-dimensional Moyal space. Its action [13] is given by

$$
S[\phi]=\int d^{4} x\left(-\frac{1}{2} \phi(-\Delta) \phi+\frac{\Omega^{2}}{2} \tilde{x}^{2} \phi^{2}+\frac{1}{2} m^{2} \phi^{2}+\frac{\lambda}{4 !} \phi \star \phi \star \phi \star \phi\right)(x)
$$

with $\tilde{x}_{\mu}=2\left(\Theta^{-1} x\right)_{\mu}$. This model has been shown renormalizable to all orders of perturbation, using different field theoretical or algebraic methods.

Several field theoretical properties have been adapted to this type of noncommutative models (see [18], [28] , [30], [29], [16], [10] and references within). Some algebraic geometrical properties of the parametric representation of the GrosseWulkenhaar model have been proved in [1].

Let us now give an elementary definition for what a primitive element in a Hopf algebra of graphs should mean for a physicist:

Definition 2.2. A primitive divergent graph of a quantum field theoretical model is a graph whose Feynman amplitude is divergent but which does not contain any subgraph for whom the Feynman amplitude is also divergent.

Working for example in position space, one can prove that, for the GrosseWulkenhaar-like models, multiplying the graph amplitude with the external fields $\phi\left(x_{i}\right)$ and integrating against these positions $x_{i}$ leads to a divergent result for the planar regular 2- and 4-point graphs. This comes from the fact that, when doing so, one takes into account contributions from "exceptional" configurations (namely for the 4-point function, the four points $x_{i}$ form a parallelogram) and generic ones. It is these "exceptional" configurations which lead to a divergent result (see [15] for the general analysis or Section 3.4 for some explicit 1-loop examples).

When working in position space, we consider in this paper graphs integrated against the positions $x_{i}$ (see again Section 3.4).

A Hopf algebra structure adapted for this noncommutative renormalization was defined in [33]. The main idea is that the notion of locality, crucial in commutative field theory, is replaced by a new one, of "Moyality", stating that the counterterms will have the same, non-local "Moyal" form as the terms in the original actions. For a more detailed discussion on this aspect the interested reader is referred to [2].

For the sake of completeness let us mention here that a noncommutative version of the Gross-Neveu model was also proved to be renormalizable at any order in 
perturbation theory [34]. Different attempts for generalizing the Grosse-Wulkenhaar idea to a gauge model have then been made and intensively studied [11].

2.3. A translation-invariant scalar model. Note that the Grosse-Wulkenhaar model (2.2) is manifestly not translation-invariant. In order to preserve the translationinvariance, one possibility is to modify the propagation in a different way ([14]):

$$
S[\phi]=\int d^{4} p\left(\frac{1}{2} p_{\mu} \phi p^{\mu} \phi+\frac{1}{2} m^{2} \phi \phi+\frac{1}{2} a \frac{1}{\theta^{2} p^{2}} \phi \phi+V^{\star}[\phi]\right),
$$

where $a$ some dimensionless parameter and $V^{\star}[\phi]$ is the corresponding potential in momentum space. The propagator is

$$
\frac{1}{p^{2}+m^{2}+\frac{a}{\theta^{2} p^{2}}} .
$$

One further chooses $a \geq 0$ such that the propagator (2.4) is positively defined. In [14], this model was proved to be renormalizable at any order in perturbation theory. Furthermore, its renormalization group flows [2] and parametric representation [31] were implemented; a mechanism for taking the commutative limit has been proposed [25] (for a review on all these developments, see [32]). Also, a propagator (2.4) like above has appeared in recent work on non-abelian gauge theory in the context of the Gribov-Zwanziger result [12]. A connection between those results and a suitable noncommutative model is unknown though at the time of writing.

Let us end this section by mentioning that propositions for noncommutative translation-invariant gauge models have been made and investigated in detail [4].

\section{Hopf algebra for the noncommutative model (2.3). Planar irregular graphs}

3.1. Considerations on its primitive divergent graphs. As proved in [14], the primitive divergent graphs of the translation-invariant model (2.3) are again the 2and 4-point graphs. However, a more thorough discussion is requested here. In the case of the planar regular graphs, these 2- and 4-point graphs will lead to the renormalization of the mass, field strength and coupling constant, just like in the case of the commutative $\phi^{4}$ model. A more tricky situation appears for the planar irregular graphs. The 4-point function graphs are proved to be convergent. The 2point function graphs (which are the ones encoding the UV/IR mixing) are again convergent. Nevertheless, when going in the UV regime of their internal momenta, they lead a priori to a $1 / p^{2}$ contribution to the respective Feynman amplitude ( $p$ being the external momenta of the respective 2-point graph). However, the modification of the propagation given in (2.3) will insure the renormalizability of the model. These 2point planar irregular graphs will just lead to a finite renormalization for the coefficient $a$ in the action (2.3). 
When inserting these 2-point graphs into bigger graphs, the latter become nonplanar (for example, when inserting the tadpole of Fig. 2 in any planar ribbon graph, the resulting ribbon graph is non-planar). In the case of the "naive" model (2.1), the Feynman amplitudes of these graphs is UV convergent but IR divergent (because of the UV/IR mixing). In the case of the model (2.3), these non-planar graphs are also convergent in IR regime, thanks to the $1 / p^{2}$ terms in the propagator (see again the proofs of [14] or [31]).

Let us conclude by stating that, for the reasons explained above, the primitive divergent graphs of the model (2.3) are taken to be the planar regular 2- and 4-point graphs. See also Section 3.5 below.

3.2. Insertions of graphs; the pre-Lie and Lie structures. In this section we explain the operation of insertion of graphs and the difficulties encountered when doing this for the ribbon graphs of NCQFT. These difficulties come from the fact that one has to deal with a non-local vertex with restricted symmetry (see Fig. 1). This insertion operations allows us to define the pre-Lie structure of Feynman ribbon graphs.

Definition 3.1. The residue res of a ribbon graph is the graph obtained by shrinking all its internal edges.

Note that the edges of any 4-point ribbon vertex present a cyclic ordering. Furthermore, for a regular graph, its external edges also define a cyclic ordering on the distinguished face containing the external edges.

Let the set of residues be (if we want to distinguish mass and wave function renormalization explicitly, we have to label edges accordingly for these external structures [8] without essential changes to our set-up)

$$
R=\{\ldots, \backslash\} \text {. }
$$

The insertion operation is defined as the bilinear map

$$
\Gamma_{1} \circ \Gamma_{2}:=\sum_{\Gamma} n\left(\Gamma_{1}, \Gamma_{2}, \Gamma\right) \Gamma .
$$

The coefficient $n\left(\Gamma_{1}, \Gamma_{2}, \Gamma\right)$ counts the number of ways to shrink $\Gamma_{2}$ to its residue in the graph $\Gamma$ such that $\Gamma_{1}$ is obtained.

Let us first deal with the insertions of planar regular graphs. We consider here insertions of a 4-point function, the 2-point function insertions being easier and thus left to the reader.

Since the graph to be inserted is considered regular, one has all the external edges breaking the same (external) face. We can thus define the afore-mentioned cyclic ordering on these external edges (see Fig. 3). One can then establish a bijection 


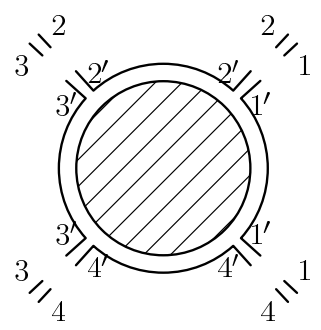

Figure 3. A 4-point planar regular graph to be inserted in some Moyal vertex. Since all the external edges are on the same face (the external) one, we can define a cyclic ordering.

between these external edges and the edges of the Moyal vertex where the insertion will be made (the gluing data, see Fig. 4).

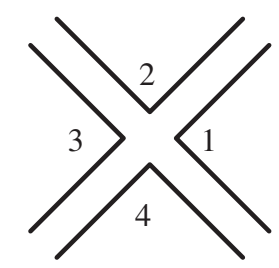

Figure 4. The Moyal vertex where the insertion is made has a cyclic ordering symmetry of its edges. One can thus realize the bijection with the external edges of the graphs to be inserted.

This gluing data can either

(1) respect the cyclic ordering (see Fig. 5) or

(2) do not respect the cyclic ordering (see Fig. 6)

We now denote by

$$
\Gamma_{1} \circ_{c} \Gamma_{2}:=\sum_{\Gamma} n\left(\Gamma_{1}, \Gamma_{2}, \Gamma\right) \Gamma
$$

the insertion as defined in (3.1). The sum on the right-hand side is always over regular graphs, and hence only insertions which respect the cycling ordering contribute.

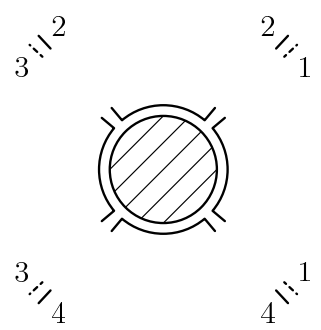

Figure 5. Insertion of a 4-point planar regular graph with gluing data respecting the cyclic ordering. 


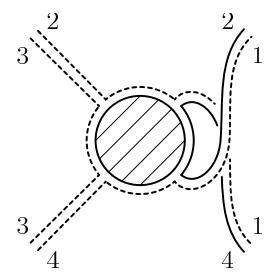

Figure 6. Insertion of a 4-point planar regular graph with gluing data not respecting the cyclic ordering.

Let us illustrate all this by working out the explicit example of Fig. 7.

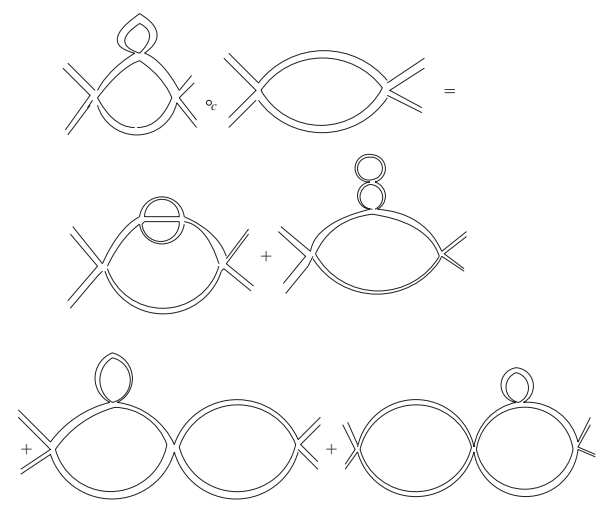

Figure 7. Example of a ribbon graph insertion which respects the cyclic ordering.

One can also consider some gluing data not respecting this cyclic ordering (as in Fig. 6). The result is shown in Fig. 8. Note that in the case of commutative $\phi^{4}$ theory the first graph on the right-hand side of Fig. 7 and the graph of Fig. 8 are equivalent. Indeed, one can use the symmetry under permutation of the incoming/outgoing fields of the local vertex to rewind the non-planar graph of Fig. 8 to the planar regular one of Fig. 7. This is not allowed in NCQFT because of the restricted symmetry of the Moyal vertex.

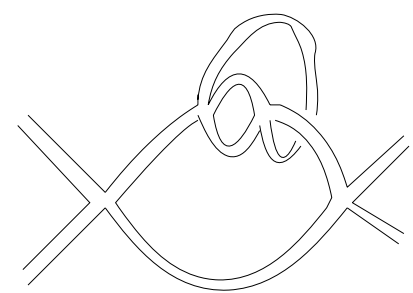

Figure 8. Result of the insertion of Fig. 7 if one uses some gluing data not respecting the cyclic ordering. 
Furthermore, in NCQFT another type of insertion is possible: one can insert a 4-point graph which is planar irregular. Not having all its external edges on the same face, one cannot define anymore some cyclic ordering on them. One can insert such a graph in a Moyal vertex in a way that reduces the number of broken faces and does not increase the genus of the resulting graph. This becomes clear in the example of Fig. 9. Inserting planar irregular graphs into planar graphs in a way that a planar (regular or

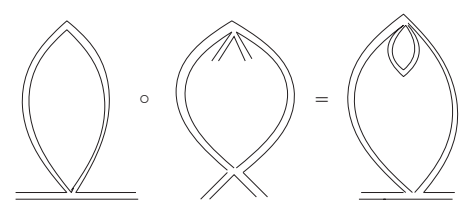

Figure 9. An example of an insertion of a planar irregular graph leading to a planar regular graph. The number of broken faces is thus reduced. We have inserted a planar irregular (thus convergent) graph in some planar regular one. The result is again planar regular.

irregular) graph is obtained is also possible for 4-point graphs with 3 broken faces, in a similar way. This is still possible because one has two legs on a particular face.

Nevertheless this is no longer possible for 4-point planar irregular graphs with 4 faces broken by external legs (consider for example the graph of Fig. 10). This

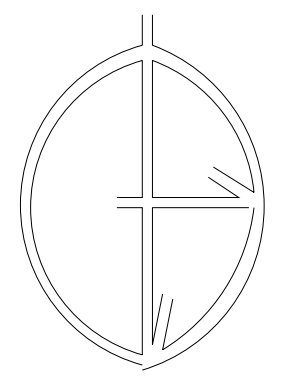

Figure 10. An example of 4-point graph with four faces broken by external legs. Each face is broken by a single line. For this reason, when inserting such a graph in some Moyal vertex, the resulting graph is non-planar.

comes from the fact that one does not have anymore two legs on the same face, whose presence, by a properly defined gluing data, could have prevented the final graph to become non-planar. One can thus conclude that in any way such a graph is inserted into a Moyal vertex the genus of the resulting graph increases.

As already mentioned in Section 3.1, the same situation occurs when one inserts a planar irregular 2-point graph (like for example the non-planar tadpole graph of Fig. 2) into some planar graphs.

We will come back to this important issue in the sequel. Note that such phenomena are irrelevant in commutative theories, as explained above. 
We close this section by a short remark on the accompanying Lie algebras. Considering the gluing data compatible with the cyclic ordering, one has an obvious pre-Lie algebra structure. Antisymmetrizing the pre-Lie product gives a Lie bracket

$$
\left[\Gamma_{1}, \Gamma_{2}\right]=\Gamma_{1} \circ_{c} \Gamma_{2}-\Gamma_{2} \circ_{c} \Gamma_{1},
$$

which defines a Lie algebra structure $L$. Consider now the graded dual of the universal enveloping algebra of this Lie structure. This gives the renormalization Hopf algebra, an algebra which is described in Section 3.3. The fact that the products (3.1) and (3.2) are indeed pre-Lie and Lie products, respectively, can thus be seen as a direct consequence of the existence of the Hopf algebra structure described in the following section.

3.3. Definition of ribbon graph Hopf algebras. Let now the unital associative algebra freely generated by 1PI non-commutative Feynman graphs (including the empty set, which we denote by $1 \mathscr{H}$ ). The product $m$ is bilinear, commutative and given by the operation of disjoint union.

We first define a core Hopf algebra $\mathscr{H}_{1 \mathrm{PI}}$, which is a straightforward generalization of the core Hopf algebra defined for a commutative theory in [5]. See [21], [22] for further details. The coproduct is defined by

$$
\Delta: \mathscr{H} \rightarrow \mathscr{H} \otimes \mathscr{H}, \quad \Delta \Gamma=\Gamma \otimes 1 \mathscr{H}+1 \mathscr{H} \otimes \Gamma+\sum_{\gamma \subset \Gamma 1 \mathrm{PI}} \gamma \otimes \Gamma / \gamma,
$$

for all $\Gamma \in \mathscr{H}_{1 \mathrm{PI}}$. The definitions of the counit and antipode follow directly and one can easily check that $\mathscr{H}_{1 \mathrm{PI}}$ is a Hopf algebra with all its cohomological richness (see for example [5], [22]).

We then define the renormalization Hopf algebra $\mathscr{H}$ as follows. In position space, let $A$ the area of the geometric figure formed by the external points $x_{1}, x_{2}, \ldots$ (For a graph with two external legs, such an area is obviously non-existent, $A=0$.) Let now a specified graph be the pair $(\Gamma, A)$. We define the coproduct

$$
\begin{aligned}
\left(\gamma, A^{\prime}\right) \otimes(\Gamma / \gamma, A), \Delta(\Gamma, A)= & (\Gamma, A) \otimes 1 \\
& +\sum_{\substack{\gamma \text { planar, regular } \\
\text { or irregular }}} n(\gamma, \Gamma / \gamma, \Gamma)\left(\gamma, A^{\prime}\right) \otimes(\Gamma / \gamma, A) .
\end{aligned}
$$

Here, in the first form, the sum runs over proper subsets of $\Gamma$ (2- or 4-point graphs) which form disjoint unions of 1PI planar graphs, and over all distinct disjoint unions of 1PI planar graphs (2- or 4-point ones) in the second form. The section coefficient $n(\gamma, \Gamma / \gamma, \Gamma)$ coincides with the one we had before.

In the case of a 4-point function, $A$ is the area of the parallelogram formed by the external points $x_{1}, \ldots, x_{4}$ (see Section 3.4). Moreover, note the appearance of the same area $A$ on the right-hand side, while the area $A^{\prime}$ is simply determined by the external leg structure of $\gamma$. Let us also remark that this construction is similar to the 
one which takes into account external structures in a commutative field theory (see [8]), thus also justifying the presence of the area $A^{\prime}$ on the left-hand side.

Let us now show how the renormalization conditions write as a function of this area. Firstly, recall that in a commutative theory, when one uses the BPHZ scheme, the renormalization condition in momentum space for the 4-point function writes

$$
G^{(4)}(0,0,0,0)=-\lambda_{\mathrm{r}},
$$

where by $r$ we mean renormalized (see for example [27]).

Let us emphasize that in perturbation theory one defines the low scales as the momentum range where the denominator of the propagator $\left(p^{2}+m^{2}\right.$ in a commutative model) is small. Thus $p=0$ is the value which minimizes this denominator. In the noncommutative theories presented in the previous section, this denominator is qualitatively changed and thus the notion of scale is also modified. We denote by $\tilde{p}$ the value of the momentum which minimizes this new type of denominator. An important fact that has to remarked is that $\tilde{p}$ is now non-vanishing.

The renormalization condition (3.5) thus becomes

$$
G^{(4)}\left(\tilde{p}_{1}, \tilde{p}_{2}, \tilde{p}_{3}, \tilde{p}_{4}\right)=-\lambda_{\mathrm{r}} e^{-\frac{i}{2} \sum_{a<b} \tilde{p}_{a}^{\mu} \Theta_{\mu \nu} \tilde{p}_{b}^{v},}
$$

where $\tilde{p}_{1}, \ldots, \tilde{p}_{4}$ are the external momenta of the graph, taken to have the same order of magnitude as $\tilde{p}$. The oscillating phase on the right-hand side of the renormalization condition (3.6) corresponds to the area associated to the respective graph. Indeed, the quartic interaction with Moyal product is invariant under the Langmann-Szabo duality [24], symmetry between momentum and direct space. Thus, in position space the oscillation on the right-hand side is proportional to the area of the respective parallelogram.

For the sake of completeness, let us also state that the 2-point function renormalization conditions are identical to the commutative ones since, as already stated above, we do not have to deal with the supplementary notion of area. The only difference comes again from the replacing of the vanishing momentum with the non-vanishing $\tilde{p}$ value.

In the rest of the paper, in order to simplify the notations, we will not further keep track explicitly of the areas $A$ or $A^{\prime}$.

The coproduct defined here allows for irregular subgraphs on the left. It is thus possible to take account of the finite renormalizations which come with such subgraphs. For the renormalization of the proper divergent graphs in our theory it suffices to divide by an (co)-ideal which eliminates irregular graphs as described below.

In the commutative case, the core Hopf algebra $\mathscr{H}_{1 \mathrm{PI}}$ contains the renormalization Hopf algebra $\mathscr{H}$ as a quotient algebra (see [5], [21]). This holds similarly here, before and after division by that (co)-ideal.

Furthermore, let us remark that the renormalization coproduct (3.4) is conceptually different from the core coproduct (3.3), both in the commutative or the noncommutative case. One can find (ribbon) graphs which have non-trivial coproducts in the 
core Hopf algebra and which are in the same time primitive elements in the renormalization Hopf algebra. Indeed, the core Hopf algebra stores more information than the renormalization one (for a more detailed analysis of this aspect in the commutative setting, the interested reader is again referred to [5], [21]).

Now let the counit be

$$
\varepsilon: \mathscr{H} \rightarrow \mathbb{K}, \quad \varepsilon\left(1_{\mathscr{H}}\right)=1, \varepsilon(\Gamma)=0,
$$

for all $\Gamma \neq 1 \mathscr{H}$. Finally the antipode is given recursively by

$$
S: \mathscr{H} \rightarrow \mathscr{H}, \quad S\left(1_{\mathscr{H}}\right)=1_{\mathscr{H}}, S(\Gamma)=-\Gamma-\sum_{\gamma} S(\gamma) \Gamma / \gamma,
$$

with the sum taken from the definition of the coproduct.

Let us emphasize that the factorization phenomena appearing in the definition (3.4) of the coproduct $\Delta$ corresponds to the renormalizability proved in [14].

We can thus state the main result of this section:

Theorem 3.2. The pair $(\mathcal{H}, \Delta)$ is a Hopf algebra.

We call ker $\varepsilon$ the augmentation ideal. Note that the quantum world, i.e., all graphs containing loops, belong to the augmentation ideal.

Let the projection

$$
P: \mathscr{H} \rightarrow \operatorname{ker} \varepsilon, \quad P=\mathrm{id}-1_{\mathscr{H}} \varepsilon
$$

and

$$
\operatorname{aug}^{\otimes k}=(P \otimes P \cdots \otimes P) \Delta^{k-1} .
$$

We define

$$
|\Gamma|_{\text {aug }}
$$

to be the augmentation degree.

Let us also denote by

the number of independent loops of some graph $\Gamma$.

The 1PI graphs $\Gamma$ provide the linear generators $\delta_{\Gamma}$. The Hopf algebra is an algebra, the free commutative (but not co-commutative) algebra of these generators. We write

$$
\mathcal{H}_{\text {lin }}=\operatorname{span}\left(\delta_{\Gamma}\right) .
$$

Note that, as in the commutative case, one can also define a Hopf algebra $\mathscr{H}_{\mathrm{rt}}$ of decorated rooted trees (see for example [3] or [20]). Furthermore, as explained in [33] the formal definition of the Bogolyubov subtraction operator remains the same as in the commutative field theoretical setting. 
3.4. More on "Moyality" or non-local renormalization. We give now some additional explanations on the coproduct rule of Section 3.3. The Moyal vertex is no longer local but is parallelogram-shaped. Using the definition of the Moyal product, the $\phi^{\star 4}$ vertex writes

$$
\begin{aligned}
\int d^{4} x \phi^{\star 4}(x) & =\int \prod_{i=1}^{4} d^{4} x_{i} \phi\left(x_{i}\right) \delta\left(x_{1}-x_{2}+x_{3}-x_{4}\right) e^{l\left(x_{1}-x_{2}\right) \wedge\left(x_{3}-x_{4}\right)} \\
& =\int \prod_{i=1}^{4} d^{4} x_{i} \phi\left(x_{i}\right) \delta\left(x_{1}-x_{2}+x_{3}-x_{4}\right) e^{l\left(x_{1} \wedge x_{2}+x_{3} \wedge x_{4}\right)},
\end{aligned}
$$

where $x \wedge y=2 x \Theta^{-1} y$. As already stated in Section 3.3, the oscillation is proportional to the area of the parallelogram.

The propagator of the Grosse-Wulkenhaar model (2.2) writes

$$
C(x, y)=\frac{1}{(2 \pi)^{2}} \int_{0}^{\infty} d \alpha \frac{\tilde{\Omega}}{\sinh ^{2} \alpha} e^{-\frac{\tilde{\Omega}}{2} \frac{(x-y)^{2}}{\tanh \alpha}-\frac{\tilde{\Omega}}{2} \tanh \alpha(x+y)^{2}-m^{2} \alpha},
$$

where $\widetilde{\Omega}=2 \theta^{-1} \Omega$. Note that propagator has the same type of exponential decay in $(x-y)$ as the commutative propagator since in the short-distance regime $\sinh \alpha$ and $\tanh \alpha$ behaves like $\alpha$. Moreover, the term in $(x+y)$ is the one responsible for the Grosse-Wulkenhaar breaking of translation-invariance. One can further define the short and long variables

$$
u=y-x, \quad v=y+x
$$

such that the propagator (3.8) rewrites

$$
C(u, v)=\frac{1}{(2 \pi)^{2}} \int_{0}^{\infty} d \alpha \frac{\tilde{\Omega}}{\sinh ^{2} \alpha} e^{-\frac{\tilde{\Omega}}{2} \frac{u^{2}}{\tanh \alpha}-\frac{\tilde{\Omega}}{2} \tanh \alpha v^{2}-m^{2} \alpha} .
$$

Let us now consider into detail the behaviour of a planar 4-point function. We focus on the example of the 1-loop graph of Fig. 11 (where we use a non-local vertex representation instead of the ribbon graph representation used in the rest of the paper) and we follow [15], where a demonstration at any order in perturbation theory has been given.

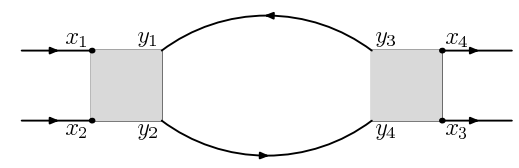

Figure 11. A planar regular 4-point graph in the Moyal $\phi^{4}$ model. 
The Feynman amplitude to analyze writes

$$
\begin{aligned}
\int d x_{1} \ldots d x_{4} d y_{1} \ldots d y_{4} \phi\left(x_{1}\right) \ldots \phi\left(x_{4}\right) \\
\quad \delta\left(x_{1}-x_{2}+y_{2}-y_{1}\right) \delta\left(y_{3}-y_{4}+x_{3}-x_{4}\right) \\
\quad C\left(y_{1}, y_{3}\right) C\left(y_{2}, y_{4}\right) e^{l\left(x_{1} \wedge x_{2}+y_{2} \wedge y_{1}\right)} e^{l\left(y_{3} \wedge y_{4}+x_{3} \wedge x_{4}\right)},
\end{aligned}
$$

where we have used the second form of (3.7) to express the Moyal oscillating phase of the two vertices. Note that, as in the commutative case, the integral above has to be considered in the short-distance (high momenta) regime for the internal lines. As above, we introduce the set of short and long variables associated to the graph:

$$
u_{1}=y_{3}-y_{1}, \quad v_{1}=y_{3}+y_{1}, \quad u_{2}=y_{2}-y_{4}, \quad v_{2}=y_{4}+y_{2} \text {. }
$$

Conversely, this writes

$$
y_{1}=\frac{1}{2}\left(v_{1}-u_{1}\right), \quad y_{2}=\frac{1}{2}\left(v_{2}+u_{2}\right), \quad y_{3}=\frac{1}{2}\left(u_{1}+v_{1}\right), \quad y_{4}=\frac{1}{2}\left(v_{2}-u_{2}\right) .
$$

We perform the change of variables

$$
\left(y_{1}, \ldots, y_{4}\right) \rightarrow\left(u_{1}, v_{1}, u_{2}, v_{2}\right)
$$

and integrate over $v_{1}$ thanks to the first of the $\delta$-functions in (3.10). After some algebra, the Feynman amplitude becomes

$$
\begin{aligned}
& \int d x_{1} \ldots d x_{4} d u_{1} d u_{2} d v_{2} \phi\left(x_{1}\right) \ldots \phi\left(x_{4}\right) \delta\left(x_{1}-x_{2}+x_{3}-x_{4}+u_{1}+u_{2}\right) \\
& C\left(u_{1}, 2\left(x_{1}-x_{2}\right)+u_{1}+u_{2}+v_{2}\right) C\left(u_{2}, v_{2}\right) e^{l\left(x_{1} \wedge x_{2}+x_{3} \wedge x_{4}\right)} \\
& e^{\left.l\left(\frac{1}{2}\left(x_{2}-x_{1}\right) \wedge u_{2}-\frac{1}{4} u_{1} \wedge u_{2}-\frac{1}{4} u_{1} \wedge v_{2}+\frac{1}{2} u_{2} \wedge v_{2}\right)\right)}
\end{aligned}
$$

where we have dropped the inessential constant coming from the change of variable (3.11).

The amplitude rewrites as

$$
\begin{gathered}
\int d x_{1} \ldots d x_{4} d u_{1} d u_{2} d v_{2} \phi\left(x_{1}\right) \ldots \phi\left(x_{4}\right) \delta\left(x_{1}-x_{2}+x_{3}-x_{4}+t\left(u_{1}+u_{2}\right)\right) \\
C\left(u_{1}, 2 t\left(x_{1}-x_{2}\right)+u_{1}+u_{2}+v_{2}\right) C\left(u_{2}, v_{2}\right) e^{l\left(x_{1} \wedge x_{2}+x_{3} \wedge x_{4}\right)} \\
\left.e^{\left.l\left(\frac{1}{2} t\left(x_{2}-x_{1}\right) \wedge u_{2}-\frac{1}{4} u_{1} \wedge u_{2}-\frac{1}{4} u_{1} \wedge v_{2}+\frac{1}{2} u_{2} \wedge v_{2}\right)\right)}\right|_{t=1} .
\end{gathered}
$$

This formula is designed such that at $t=0$ all dependence on the external variables $x$ factorizes out of the $u, v$ integral, giving the desired vertex form. One has then to perform a Taylor expansion with respect to the $t$ variable: $f(1)=f(0)+\int_{0}^{1} d t f^{\prime}(t)$. The first term, $f(0)$, is of the desired parallelogram-like form; furthermore, the remainder term is proved to be irrelevant (see [15] for details). 
This is thus a straightforward generalization of the commutative situation, where one has replaced the notion of locality of the vertex with the more involved notion of non-local but parallelogram-shaped vertex of a Moyal field theory. The same type of arguments also holds for the model (2.3) and so justifies the choice of coproduct made in Section 3.3.

Let us now give some insights on the planar irregular case in order to better understand why these graphs are finite and thus are not primitively divergent (and hence do not have to be taken into consideration when defining the coproduct). As before, we work in position space and we consider the example of an 1-loop graph, namely the one in Fig. 12. With the conventions detailed above, the Feynman amplitude to

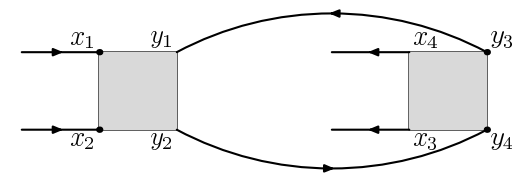

Figure 12. A planar irregular 4-point graph in the Moyal $\phi^{4}$ model.

analyze writes

$$
\begin{aligned}
& \int d x_{1} \ldots d x_{4} d y_{1} \ldots d y_{4} \phi\left(x_{1}\right) \ldots \phi\left(x_{4}\right) \delta\left(x_{1}-x_{2}+y_{2}-y_{1}\right) \\
& \quad \delta\left(x_{4}-y_{4}+y_{3}-x_{3}\right) C\left(y_{1}, y_{3}\right) C\left(y_{2}, y_{4}\right) e^{l\left(x_{1} \wedge x_{2}+y_{2} \wedge y_{1}\right)} e^{l\left(x_{4} \wedge y_{4}+y_{3} \wedge x_{3}\right)} .
\end{aligned}
$$

We perform the same change of variable (3.11) as in the planar regular case; as above, we integrate over $v_{1}$ (using the first of the $\delta$-functions in (3.13)). We then focus on the variables $x_{4}$ and $v_{2}$ which are the ones leading to an improved behaviour with respect to the planar regular case (3.12). Thus, the factor involving these variables in the oscillation in the final form of the amplitude is

$$
e^{l\left(-v_{2} \wedge x_{4}+\frac{1}{2} v_{2} \wedge\left(x_{3}+x_{1}-x_{2}\right)\right)} .
$$

This factor is different of the one found in (3.12) and when integrated against $x_{4}$ and $v_{2}$ (taken into account the $v_{2}$ contribution of the propagators (3.9)) leads to an improvement in the UV behaviour of the integral (see again [15] for details). Thus, the Feynman integral (3.12), logarithmically divergent in the planar regular case, becomes convergent, as announced above.

Note that this mechanism is much simpler in momentum space, where one just has, in the planar irregular case, a supplementary oscillation factor which makes convergent the (initially logarithmically divergent) integral.

To end this section, let us conclude that the "Moyality" principle relies on gluing parallelograms together. The result is a new parallelogram whose area is the sum of the two initial parallelograms if and only if the respective graph is planar regular (see 
Fig. 13 and 14, where we use again a non-local vertex representation). The remarkable thing is that power counting requires renormalization only for these graphs!

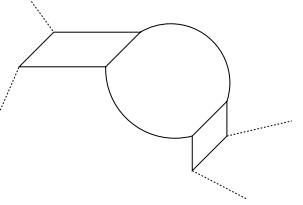

Figure 13. A 4-point graph in the noncommutative $\phi^{\star 4}$ model. The point-like vertices now become parallelogram-shaped vertices (in position space). The Moyal vertex also has an oscillation proportional to the parallelogram area.

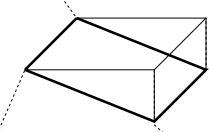

Figure 14. When considering the short-distance (high energy) regime the two initial parallelograms form a parallelogram whose area is the sum of the initial parallelograms. This is true if and only if the graph is planar. This illustrates the principle of "Moyality".

Let us also stress here that the proof of "Moyality" in the algebraic language used in this paper is given in Section 5, where appropriate Hochschild 1-cocyles are defined.

3.5. Planar irregular graphs; semi-direct structure. In this section we come back on the issue of the 2-point planar irregular graphs, this time from the point of view of the Hopf algebra defined in the previous section.

Proposition 3.3. The ideal $\mathscr{H}^{\mathrm{pli}}$ generated by the 1PI 2-point planar irregular Feynman graphs is a Hopf ideal and coideal in $\mathcal{H}$,

$$
\Delta\left(\mathscr{H}^{\mathrm{pli}}\right) \subseteq \mathscr{H}^{\mathrm{pli}} \otimes \mathscr{H}+\mathscr{H} \otimes \mathscr{H}^{\mathrm{pli}}, \quad \varepsilon\left(\mathscr{H}^{\mathrm{pli}}\right)=0, S\left(\mathscr{H}^{\mathrm{pli}}\right) \subseteq \mathscr{H}^{\mathrm{pli}} .
$$

Proof. Let us consider the non-trivial part of the coproduct $\Delta$. We denote the interior broken face by $f$. If one chooses some 2- or 4-point subgraph of $\Gamma$ to contain the face $f$, then the respective subgraph will be planar irregular and hence not primitive divergent. Thus, all the primitive divergent subgraphs must not contain the respective face $f$. This face will therefore be retrieved in the cograph, which leads to

$$
\Delta^{\prime}\left(\mathscr{H}^{\mathrm{pli}}\right) \subseteq \mathscr{H} \otimes \mathscr{H}^{\mathrm{pli}}
$$


Taking also the trivial part of the coproduct one obtains (3.14). Note that when applying the coproduct on these ribbon graphs, the number of internal faces conserves as a sum of the number of internal faces of the graph and of the cograph.

A direct consequence of this proposition is that one can discard this planar irregular sector (as we did in the previous section) by simply taking the respective quotient by the (co)-ideal $\mathscr{H} / \mathscr{H}^{\text {pli }}$. This cannot however be done for the non-planar sector as well because the non-planar sector does not form a Hopf coideal (one can easily find some counterexamples). We can eliminate it though by working in a suitable quotient Hopf algebra.

Furthermore, let us emphasize the fact that in Proposition 3.3 above we have dealt with both the notion of Hopf coideal (implying the use of the coproduct $\Delta$ ) and the (here trivial) notion of ideal (implying the use of the product $m$ ).

We have thus established in this section three distinct Hopf algebra structures:

(1) the core Hopf algebra $\mathscr{H}_{1 \mathrm{PI}}$ given by (3.3),

(2) the Hopf algebra $\mathscr{H}$ given by (3.4),

(3) the Hopf algebra obtained from $\mathscr{H}$ by diving with the (co)-ideal eliminating the planar irregular graphs (as described above).

Let us end this section by giving the semi-direct structures of the Lie algebras associated with the three cases above.

Proposition 3.4. The Lie algebra $L$ is the semi-direct product of the abelian Lie algebra $L_{0}$ by $L_{c}$, where with respect to the three cases enumerated above one has:

(1) $L_{c}$ is $\mathscr{H}_{1 \mathrm{PI}}$ and $L_{0}$ is the empty set.

(2) $L_{c}$ is generated by the planar regular 2- and 4-point graphs, planar irregular 4-point graphs with two or three broken faces and $L_{0}$ is generated by the $6,8, \ldots$-point graphs (planar regular and irregular) as well as the 2-point planar irregular graphs and 4-point planar irregular graphs with four broken faces.

(3) $L_{c}$ is generated by the planar regular 2-and 4-point graphs, and $L_{0}$ is generated by the $6,8, \ldots$-point graphs (planar regular).

Proof. In the first case, there is no difference to the commutative case; the result stated above is a straightforward consequence of the fact that vertices of any valence are allowed to appear, as opposed to usual Feynman graphs in renormalizable perturbative quantum field theories (see [22] for more details). The second case is the most involved one. One expects to have the $L_{c}$ part generated simply by the planar regular and irregular 2- and 4-point graphs. Nevertheless, we have seen in Section 3.2 that the planar irregular 2-point graphs and the planar irregular 4-point graphs with four broken faces cannot be inserted without increasing the genus (thus leading to nonplanarity). Finally, the last case is treated along the same lines as the renormalization Hopf algebra of commutative theories (see [8]) since we deal here only with planar 
regular graphs (and with insertions respecting the cyclic ordering, as explained in Section 3.2).

\section{More on ribbon graphs}

4.1. Symmetry factor of graphs. The symmetry factor of a graph $\Gamma$ is defined as the rank of automorphism group of $\Gamma$. The use of ribbon graph changes the picture with respect to the $\phi^{4}$ theory. One has, for example a symmetry factor of 1 for the down (resp. up) tadpoles of Fig. 15 (resp. Fig. 16).

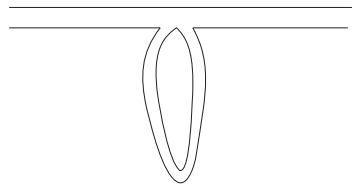

3

Figure 15. The down tadpole. This graph is planar regular and hence primitive. Its symmetry factor is equal to 1 .

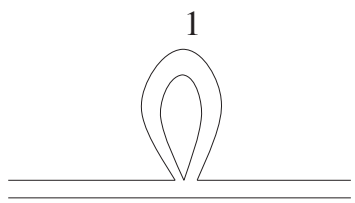

Figure 16. The up tadpole. This graph is planar regular and hence primitive.

Proposition 4.1. The symmetry factor of a ribbon graph in NCQFT is equal to 1.

Proof. We proceed by induction on the number of loops $b$. We thus consider the case $b=1$. To start with, take the planar regular 4-point graph of Fig. 17, where we have denoted, at the first vertex, the external momenta by $p_{a}$ and $p_{b}$ and the internal ones

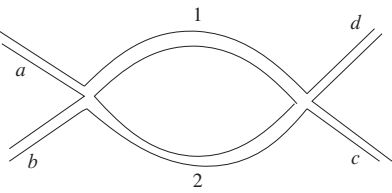

Figure 17. An example of an 1-loop ribbon graph.

by $p_{1}$ and $p_{2}$ (note the cyclic ordering at the vertex). In a commutative theory, this graph's symmetry factor is 2 since it is equivalent to the one of Fig. 18, where the 
internal momenta $p_{1}$ and $p_{2}$ have been interchanged. Nevertheless, this is no longer true in the noncommutative setting described here because the cyclic ordering at the vertex is not respected. Thus, the symmetry factor of the ribbon graphs of Fig. 17 and 18 are each equal to 1 . This results generalize in a straightforward manner for any 1-loop graph.

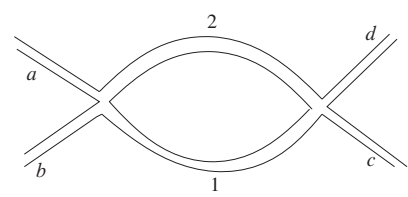

Figure 18. Another example of an 1-loop ribbon graph.

To obtain graphs with higher number of loops, we can insert 2- and resp. 4-point graphs in propagators and resp. Moyal vertices. Thus, if the statement is true for some $b \in \mathbb{N}$ it will be true for $b+1$ loops. This completes the proof.

Let us remark that this proposition is a direct consequence of the fact that the Moyal vertex is symmetric only under cyclic permutation of the incoming/outgoing fields (see above). In the case of commutative field theory it is the symmetry under the total group of permutations of the vertex that is responsible for non-unit symmetry factors.

\subsection{Permutation of external edges}

Definition 4.2. Let

$$
|\Gamma|_{V}
$$

be the number of distinct ribbon graphs $\Gamma$ which are equal upon removal of external edges.

Let us consider the example of Fig. 19. Note that in the case of commutative $\phi^{4}$ theory one has $|\Gamma|_{V}=3$ for the example above (see [19]). The missing third graph disappears because we consider only the planar regular sector of the theory.

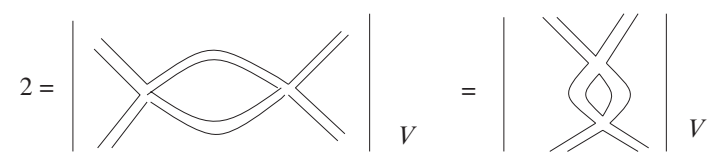

Figure 19. An example for $\Gamma_{V}$. 


\subsection{Number of maximal forests}

Definition 4.3. The number of maximal forests maxf of a graph $\Gamma$ is the number of ways to shrink subdivergencies to Moyal points such that the resulting cograph is primitive.

Let us now give an example which illustrates the difference in calculating maxmf with respect to the commutative $\phi^{4}$ graphs. The graph of Fig. 20 has a maximal number of forests equal to two. Indeed, when the subdivergence is represented by

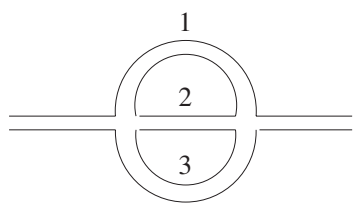

Figure 20. The sunshine graph, with three internal lines. Its maximal number of forests is equal to 2 .

the bubble graph formed of the internal lines 1 and 2 then the resulting cograph is the down tadpole of Fig. 15. If the subdivergence is taken to be the bubble graph formed of the internal lines 2 and 3 then the resulting cograph is up tadpole of Fig. 16. Finally, if one takes the divergence to be given by the internal lines 1 and 3, then the resulting cograph is the "non-planar" tadpole of Fig. 21 which is not primitive. Hence, maxf

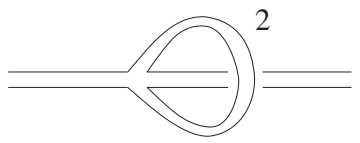

Figure 21. The "non-planar" tadpole obtained from shrinking the bubble graph formed by lines 1 and 3 in the sunshine graph of Fig. 20.

is equal to 2. In the case of the commutative $\phi^{4}$ theory, the three tadpoles above are equivalent and thus the number of maximal forests will be equal to 3 .

4.4. Number of bijections when gluing graphs; number of insertion places. We denote by $\operatorname{bij}\left(\gamma_{1}, \gamma_{2}, \gamma\right)$ the number of bijections between the external edges of $\gamma_{2}$ and adjacent edges of places $p$ res $\left(\gamma_{2}\right)$ in $\gamma_{1}$ such that $\gamma$ is obtained.

We call (subsets of) edges and vertices of a graph places of the respective graph. We also use the notation $[\gamma \mid X]$ for the number of insertion places of the graph $X$ in $\gamma$.

The values taken by this parameter do not change if one deals with ribbon graphs. Let us end this section by recalling that in [19] other parameters on Feynman graphs have been defined. All these generalize to ribbon graphs (one just needs to take care 
of the differences like the one we saw already here). We do not need in this paper these other notions, so we do not deal with them here.

\section{Hochschild cohomology in NCQFT: Moyality and Dyson-Schwinger equations}

We prove in this section that every divergent graph $\gamma$ without subdivergencies determines a Hochschild 1-cocyle $B_{+}^{\gamma}$ (see below). Furthermore, any relevant graph in the perturbative expansion is in the range of such a 1-cocyle.

We also study the Dyson-Schwinger equation as formal construction based on the Hochschild cohomology of these Hopf algebras. Thus one can state that the Hochschild cohomology leads the way from perturbative to non-perturbative physics. This construction extends the one of commutative field theories (see for example [3], [19]). Throughout this section we generally follow closely the results in [3], [20], [19].

Before going further, let us state here that these results hold for the algebraic structures associated to both types of renormalizable NCQFT models introduced in Section 2.

Let us firstly recall (following [20]) some useful definitions regarding the Hochschild cohomology. Let $A$ be a bialgebra and $\Delta$ its coproduct. We regard linear maps

$$
L: A \rightarrow A^{\otimes n}
$$

as $n$-cochains. We define a coboundary map $b$,

$$
b^{2}=0
$$

by

$$
b L:=(\mathrm{id} \otimes L) \circ \Delta+\sum_{i=1}^{n}(-1)^{i} \Delta_{i} \circ L+(-1)^{n+1} L \otimes \mathbb{1},
$$

where $\Delta_{i}$ denotes the coproduct applied to the $i$-th factor in $A^{\otimes n}$. This defines the cohomology of $A$.

Now let $\left(B_{+}^{d_{n}}\right)_{n \in \mathbb{N}}$ be a set of Hochschild 1-cocyles on such a Hopf algebra. The Dyson-Schwinger equation writes

$$
X=\mathbb{1}+\sum_{n=1}^{\infty} \omega_{n} \lambda^{n} B_{+}^{d_{n}}\left(X^{n+1}\right)
$$

in $\mathscr{H} \llbracket \lambda \rrbracket$. The parameters $\omega_{n}$ are scalars. One decomposes the solution as

$$
X=\sum_{n=0}^{\infty} \lambda^{n} c_{n} \quad \text { with } c_{n} \in \mathscr{H}
$$


One has to sum up on the contribution corresponding to the planar regular as well to the planar irregular ribbon graphs since the latter sector can lead to (planar regular) contributions when the operator $B_{+}$interferes (see Section 3.2). This is a major difference with respect to the commutative case because in a commutative framework this distinction is irrelevant and one does not has to deal with this type of phenomena. We can illustrate this by explicitly splitting

$$
c_{n}=c_{n}^{\mathrm{reg}}+\tilde{c}_{n},
$$

where by $c_{n}^{\text {reg }}$ we refer to the regular sector and by $\tilde{c}_{n}$ we refer to the irregular sector.

Lemma 5.1 ([3], Lemma 2.1, p. 150). The Dyson-Schwinger equation (5.1) has a solution given by $c_{o}=\mathbb{1}$ and

$$
c_{n}=\sum_{m=1}^{n} \omega_{m} B_{+}^{d_{m}}\left(\sum_{\substack{k_{1}+\cdots+k_{m+1}=n-m \\ k_{i} \geq 0}} c_{k_{1}} \ldots c_{k_{m+1}}\right) .
$$

Proof. As in the commutative case (see [3]), one needs to insert the ansatz (5.2) in the Dyson-Schwinger equation (5.1). Sorting then by powers in the coupling constant $\lambda$ yields the result. Furthermore, uniqueness is obvious. The use of ribbon graphs does not change the validity of these arguments. The only thing that is changed is the fact that one has to sum up also on the planar irregular sector, which leads via the operator $B_{+}$to planar regular contributions.

Let us now switch for the moment to a description in terms of decorated trees. We denote by $\operatorname{dec}(v)$ the decoration and by fert $(v)$ the fertility (i.e., the number of outgoing edges) of the vertex $v$. Furthermore, the decoration weight of such a tree is defined as the sum of the decorations of the vertices. We then define the coefficients

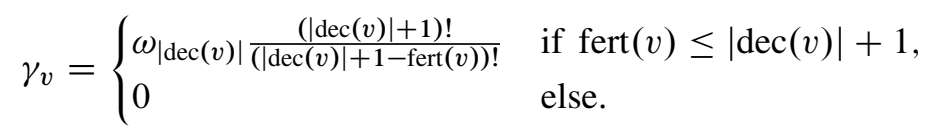

For such a decorated tree, the coefficient

$$
\prod_{v} \gamma_{v}
$$

can be interpreted by considering every decorated tree as an operadic object with $|\operatorname{dec}(v)|+1-\operatorname{fert}(v)$ inputs at each vertex $v$. The total number of inputs is $n+1$, where $n$ is the decoration weight of the respective tree. The coefficient (5.3) is the number of planar (i.e., noncommutative) embeddings of this operadic tree when keeping the trunk (i.e., the original tree) fixed. Note that the sense of "planar" and resp. "noncommutative" here refers to the decorated trees and not to the Feynman graphs or resp. spacetime (as used in the rest of the paper). For an explicit example of the interpretation above, we refer to [3]. 
This type of reasoning is not dependent on the fact that the respective decorated tree was obtained from a ribbon or an "usual" commutative Feynman graph.

Before going further let us remark that the operation of inserting graphs into graphs can be mathematically written down in an operadic language.

Theorem 5.2 ([3], Theorem 2.2, p. 150). The elements $c_{n} \in \mathscr{H}$ generate a Hopf subalgebra in $\mathscr{H}$,

$$
\Delta\left(c_{n}\right)=\sum_{m=1}^{n} P_{k}^{n} \otimes c_{n},
$$

where $P_{n}^{k}$ are polynomials of degree $n-k$ in the elements $c_{\ell}, \ell \leq n$, given by

$$
P_{k}^{n}=\sum_{l_{1}+\cdots+l_{k+1}=n-k} c_{l_{1}} \ldots c_{l_{k+1}}
$$

Proof. We give here an operadic proof which follows the one of [3]. Let $\mu_{n}$ some maps in

$$
O^{[n]}: V^{\otimes n} \rightarrow V
$$

for some space $V$ and $G(\lambda)$ a formal series in $\lambda$ with coefficients in $O^{[j]}$. We denote the identity map on $V$ as $\mathbb{1}_{V}$. As a variation of the Dyson-Schwinger equation (5.1) we write down the operadic fix point equation

$$
G(\lambda)=\mathbb{1}_{V}+\sum_{n} \lambda^{n} \mu_{n+1}\left(G(\lambda)^{\otimes(n+1)}\right) .
$$

One writes $G(\lambda)=\mathbb{1}_{V}+\sum_{k} \alpha^{k} v_{k}$. By induction it then follows that $v_{k} \in O^{[k+1]}$. Furthermore, $G(\lambda)$ is a sum (with unit weights) over all maps which one obtain by composition of some undecomposable maps $\mu_{n}$.

Let us now consider the coproduct of decorated rooted trees and some monomial

$$
v^{r_{1}} \ldots v_{i_{l}}^{r_{l}}
$$

Note that this monomial lives in the PROP $V^{\otimes\left(r_{1} i_{1}+\cdots+r_{l} i_{l}+r\right)} \rightarrow V^{\otimes r}$, where $r=$ $\sum_{i=1}^{l} r_{i}$. The number of ways such a monomial can be composed with any element in $O^{[r-1]}$ is

$$
\frac{r !}{r_{1} ! \ldots r_{l} !}
$$

This is the contribution to the term in the coproduct which has $v_{k}$ on the right-hand side and the given monomial on the left-hand side (because the $v_{i}$ sum over all maps with unit weight). The same argument (5.5) also determines the coproduct on the $c_{k}$ on the initial Dyson-Schwinger equation (5.1):

$$
P_{k}^{n}=\sum_{\substack{i_{1} r_{1}+\ldots+i_{l} r_{l}=n-k \\ 0 \leq i_{s}<i_{s}+1 \leq k \\ \sum r_{i}=k+1}} \frac{(k+1) !}{r_{1} ! \ldots r_{l} !} c_{i_{1}}^{r_{1}} \ldots c_{i_{l}}^{r_{l}} .
$$


This comes from the fact that the trees in $c_{k}$ are weighted by the noncommutative (planar) product (5.3) over the vertices; the coproduct respects this planar structure.

Equation (5.6) is in agreement with (5.4). As above, these arguments apply also in the case of NCQFT when replacing the Feynman graphs with ribbon Feynman graphs.

Remark 5.3. The coefficient (5.3) corresponds to the noncommutative (planar) case while the coefficient (5.6) corresponds to the commutative (non-planar) case. Note that, as in the proof of Theorem 5.2, the terminology "commutative" (resp. "planar") is related to the decorated rooted trees and not to spacetime (resp. Feynman graphs).

Let us come back to the case of the renormalization Hopf algebra of Feynman graphs. As in [19] we define the maps from $\mathscr{H}$ to $\mathscr{H}_{\text {lin }}$,

$$
B_{+}^{k ; r}=\sum_{\substack{|\gamma|=k \\|\gamma| \text { lag }=1 \\ \text { res }(\gamma)=r}} B_{+}^{\gamma}, \quad B_{+}^{\gamma}(X)=\sum_{\Gamma \in \mathscr{H}_{\operatorname{lin}}} \frac{\operatorname{bij}(\gamma, X, \Gamma)}{|X|_{\vee}} \frac{1}{\operatorname{maxf}(\Gamma)} \frac{1}{[\gamma \mid X]} \Gamma,
$$

for all graphs $X$ in the augmentation ideal. Furthermore, we let

$$
B_{+}^{\gamma}\left(1_{\mathscr{H}}\right)=\gamma
$$

This definition ensures that $B_{+}^{k ; r}$ is a Hochschild closed map. This is achieved thanks to the counting of the number of maximal forests. Thus the map $B_{+}^{\gamma}$ is a generalization of the pre-Lie insertion into $\gamma$ (see [19] for further details).

Let

$$
c_{k}^{r}=\sum_{\substack{|\Gamma|=k \\ \operatorname{res}(\Gamma)=r}} \Gamma
$$

be the sum of graphs of a given loop number and residue, and let $M_{r}$ be the set of graphs such that $\operatorname{res}(\Gamma)=r$ for all $r \in R$.

Following again [19], one has the following result:

Theorem 5.4 ([19], Theorem 5). (i) $\Gamma^{r} \equiv 1+\sum_{\Gamma \in M_{r}} \Gamma=1+\sum_{k=1}^{\infty} g^{k} B_{+}^{k: r}\left(X_{k, r}\right)$,

(ii) $\Delta\left(B_{+}^{k ; r}\left(X_{k, r}\right)\right)=B_{+}^{k ; r}\left(X_{k, r}\right) \otimes \mathbb{1}+\left(\mathrm{id} \otimes B_{+}^{k ; r}\right) \Delta\left(X_{k, r}\right)$,

(iii) $\Delta\left(c_{k}^{r}\right)=\sum_{j=0}^{k} \operatorname{Pol}_{j}^{r}(c) \otimes c_{k-j}^{r}$, where $\operatorname{Pol}_{j}^{r}(c)$ is a polynomial in the variables $c_{m}^{r}$ of total degree $j$.

Let us also mention the important fact that the Hochschild 1-cocyle $B_{+}$above mixes the planar irregular sector with the planar regular sector of the theory by the mechanism showed in Section 3.2. Thus, in the result (i) above, this planar irregular 
sector has to be included in the set of graphs $X_{k, r}$ of loop number $k$ and residue $r$. This is a major difference with the case of commutative field theory.

The result (iii) ensures that the elements $c_{k}^{r}$ form a Hopf subalgebra. As it was showed for a commutative field theory in [6], this is of particular importance in the road towards some exact solution of the Dyson-Schwinger equation.

\section{A two-loop example}

To illustrate the theorems of the previous section, we completely work out here a non-trivial two-loop example.

6.1. One loop. For the noncommutative propagator and vertex, one has

$$
\begin{aligned}
& B_{+}^{1, \overline{\bar{Q}}}=B_{+}^{\overline{(}}+B_{+} \\
& B_{+}^{1,}=B_{+}+B_{+} .
\end{aligned}
$$

Applying this map on the unit of the Hopf algebra of graphs $1_{\mathscr{H}}$ leads to

$$
\begin{aligned}
& c_{1}=B_{+}^{1,-}\left(1_{\mathscr{H}}\right), \\
& c_{1}=B_{+}^{1,}\left(1_{\mathscr{H}}\right) .
\end{aligned}
$$

Applying (5.8) and (6.1) leads trivially to

$$
\begin{aligned}
& c_{1}=\Longleftarrow+\bar{Q}, \\
& c_{1}=\bigcirc+X .
\end{aligned}
$$

Let us now go further to the more involved case of the two-loop computations.

6.2. Two loops. We first work out the easier two-loop two-point function and then proceed with the four-point one. Using the definition (5.9), one gets

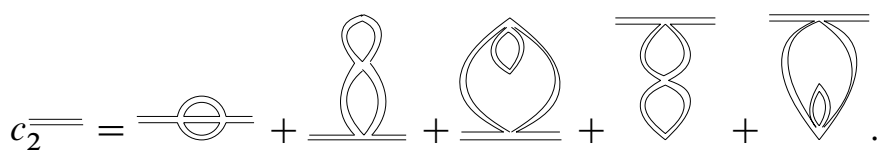


Applying the coproduct $\Delta$ on each of these ribbon graphs, one has (for the non-trivial part):

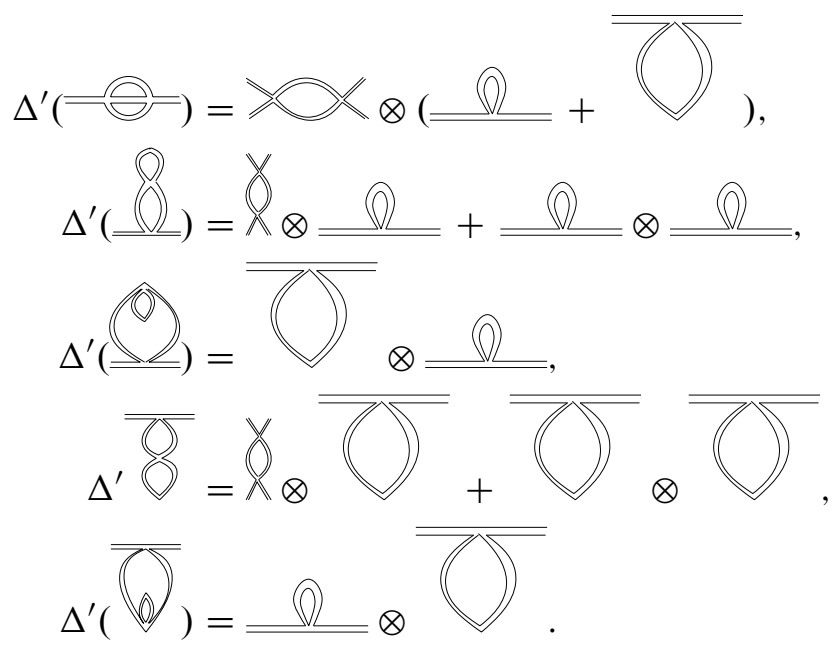

Putting all this together leads to

$$
\Delta^{\prime}\left(c_{\overline{2}}\right)=\left(c_{1}+c_{\overline{1}}\right) \otimes c_{1}^{\overline{1}} .
$$

Let us now focus on the more involved case of the four-point function. Using the definition (5.9), one gets

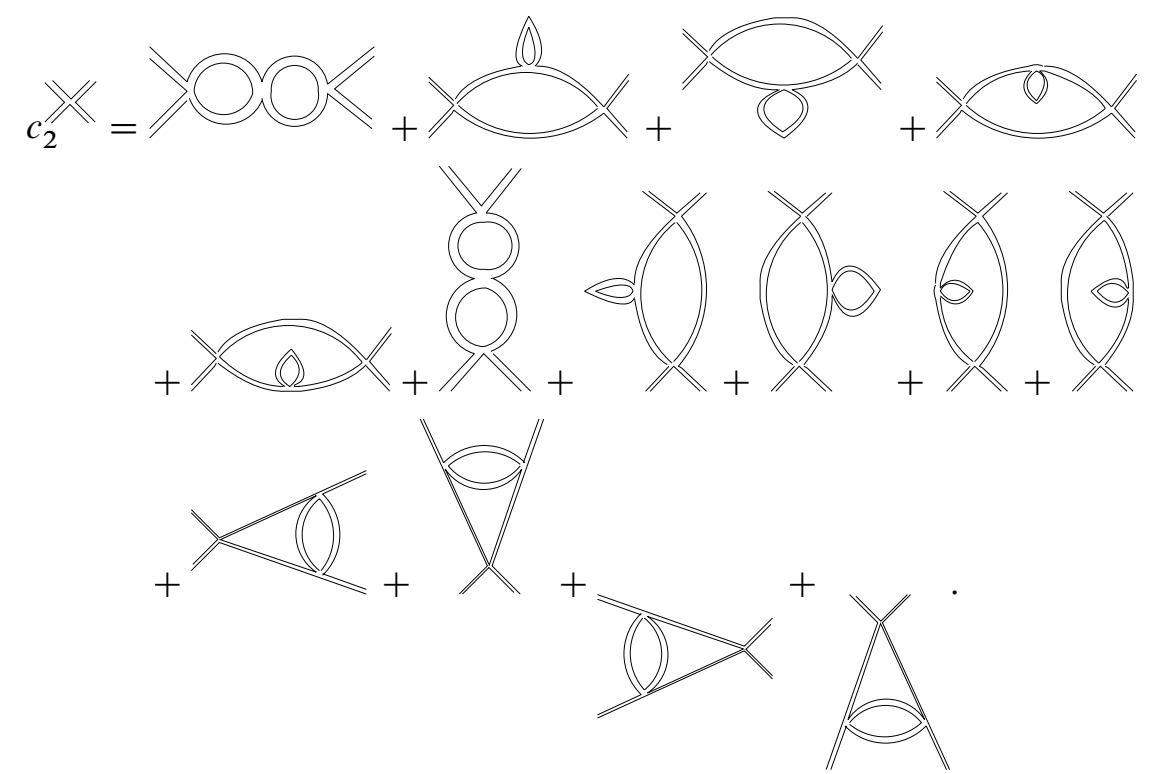

Applying the coproduct $\Delta$ on each of these ribbon graphs, one has (for the non-trivial 
part):

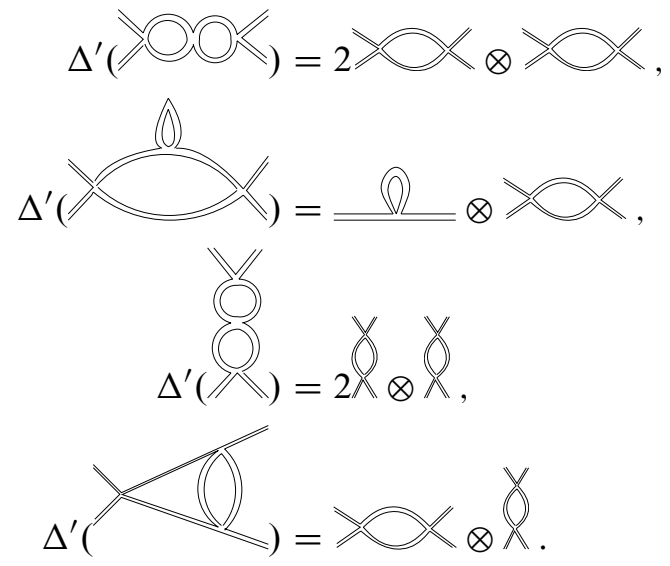

The remaining ten ribbon graphs on the right-hand side of (6.5) are treated analogously, finally leading to

$$
\Delta^{\prime}\left(c_{2}\right)=\left(2 c_{1}+2 c_{1}\right) \otimes c_{1}
$$

(where we have used (6.2)). The results (6.4) and (6.6) are thus illustrations of Theorem 5.4 (iii); these equations further give the expressions of the polynomials $P_{1} \overline{\text { and }} P_{1}$.

Let us now show that each such two-loop graph lies in the image of our Hochschild one-cocycles $B_{+}^{1,}=$. Using the definition (5.7) and computing the combinatorial factors as indicated in Section 4, one has

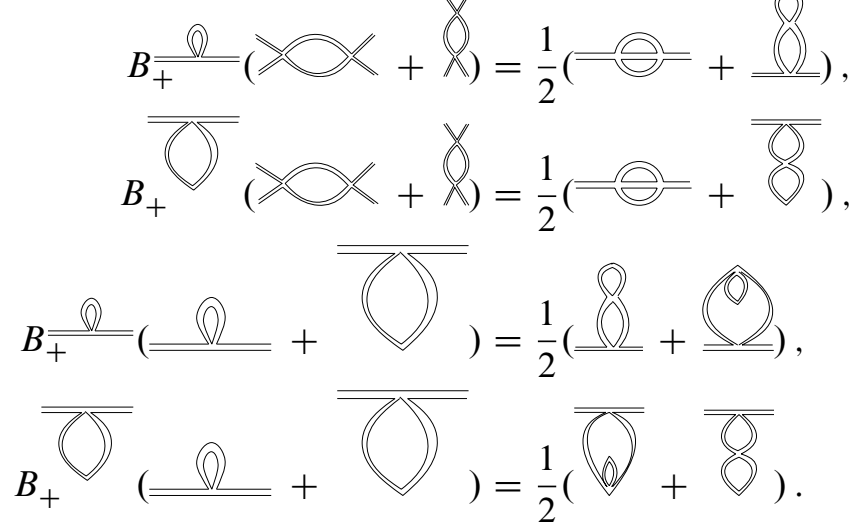

The $\frac{1}{2}$ coefficients above come from the computation of the permutation of external legs, number of bijections and number of maximal forests for each of the resulting ribbon graphs, as explained in Section 4. When adding up all this, one does not obtain 
$c \overline{\overline{2}}$ (as given by (6.3)). This comes from the fact that we have not yet included the planar irregular sector, which gives birth through these insertions to planar regular graphs (as explained in the Section 3.2). One has

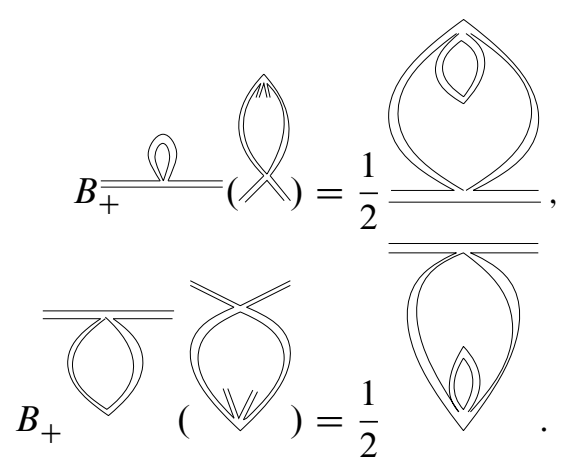

The two new graphs above belong to

$$
\tilde{c}_{1}
$$

(which corresponds to the planar irregular sector). Note that the rest of the planar irregular graphs belonging to (6.9) does not lead to planar regular graphs when acting upon with $B_{+}^{1,}=$. Furthermore, one can analogously define

$$
\tilde{c} \overline{\overline{1}}=\overline{ } .
$$

Acting on this graph with $B_{+}^{1,}=$ does not lead to a planar regular graph. Thus, adding up (6.7) and (6.8), one obtains indeed $c_{2} \overline{\overline{2}}$, as expected. We have thus proved that

$$
c \overline{\overline{2}}=B_{+}^{1, \overline{ }}\left(c_{1}^{\overline{1}}+c_{1}+\tilde{c}_{\overline{1}}^{\bar{c}}+\tilde{c}_{1}\right) .
$$

Let us now explicitly show the necessity of adding the irregular sector also when writing down Theorem 5.4 (ii) at this two-loop level. In order to do this we first consider the four planar regular graphs:
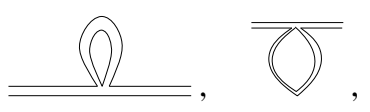

Using (6.7) and the definition (3.4) of the coproduct one can write down the left-hand side and the right-hand side of Theorem 5.4 (ii). Let us right down the contribution of the graphs of (6.11) to the left-hand side of Theorem 5.4 (ii). One has the following relations: 
(1)

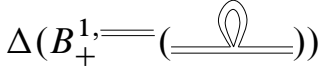

$$
\begin{aligned}
& \left.=\Delta\left(\left(B_{+}+B_{+}\right)(\underline{\underline{(\Omega}})\right)\right) \\
& =\frac{1}{2} \Delta(\underline{\underline{O}}+\infty) \\
& =\frac{1}{2}-\otimes 1_{\mathscr{H}}+\frac{1}{2} 1_{\mathscr{H}} \otimes-\frac{1}{2} \otimes \otimes+\frac{1}{2} \Perp \\
& +\frac{1}{2} \otimes 1 \mathscr{H}+\frac{1}{2} 1_{\mathscr{H}} \otimes+\frac{1}{2}=\otimes
\end{aligned}
$$

(2)

$$
\begin{aligned}
& \left.\Delta\left(B_{+}^{1,-}(\bar{Q})\right)=\Delta\left(\left(B_{+}^{\bar{Q}}+B_{+}\right)(\overline{\bar{Q}})\right)\right) \\
& =\frac{1}{2} \Delta(\circlearrowleft)+ \\
& =\frac{1}{2} \otimes 1_{\mathscr{H}}+\frac{1}{2} 1_{\mathscr{H}} \otimes+\frac{1}{2} \otimes \\
& +\frac{1}{2} \bigcirc \otimes 1_{\mathscr{H}}+\frac{1}{2} 1_{\mathscr{H}} \otimes \bigcirc+\frac{1}{2} \bigotimes \otimes \\
& +\frac{1}{2} \circlearrowright \otimes \overline{\left(\sum_{.}\right.}
\end{aligned}
$$

(3)

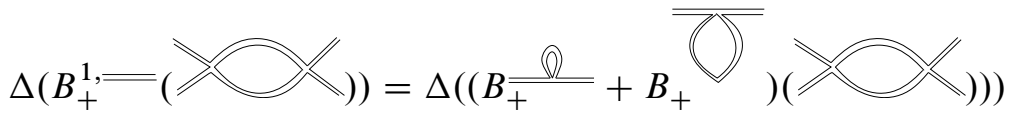

$$
\begin{aligned}
& =\Delta(\square) \\
& =\square \otimes 1_{\mathscr{H}}+1_{\mathscr{H}} \otimes \square
\end{aligned}
$$

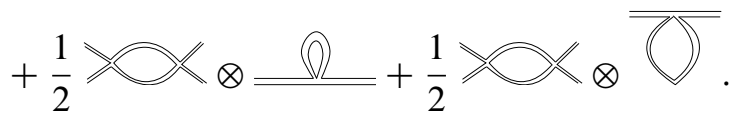


(4)

$$
\begin{aligned}
& \left.\Delta\left(B_{+}^{1,-}(\wedge)\right)=\Delta\left(\left(B_{+}^{\bar{C}}+B_{+}\right)(\lambda)\right)\right) \\
& =\frac{1}{2} \Delta(=+\bigcirc)
\end{aligned}
$$

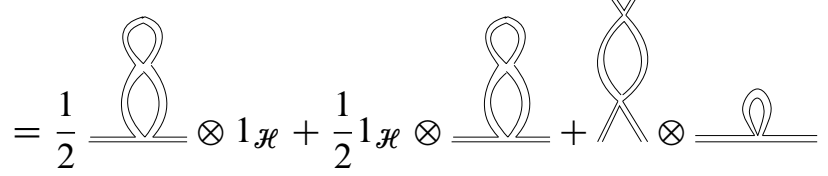

$$
\begin{aligned}
& +\frac{1}{2}=\otimes \frac{1}{2} \otimes \otimes 1_{\mathscr{H}}+\frac{1}{2} 1_{\mathscr{H}} \otimes \\
& +\widehat{N}_{\otimes}+\frac{1}{2} \bar{\nabla} \otimes \overline{\bar{O}} .
\end{aligned}
$$

Adding together the contributions of equations (6.12) to (6.13) one has for the left-hand side of Theorem 5.4 (ii) the following results (corresponding to the planar regular graphs listed in equation (6.11)):

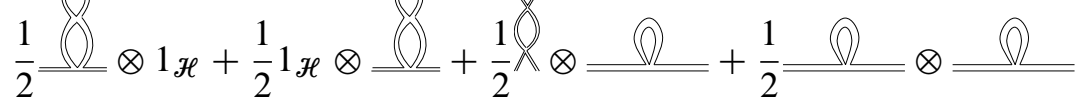

$$
\begin{aligned}
& +\frac{1}{2} \otimes 1_{\mathscr{H}}+\frac{1}{2} 1_{\mathscr{H}} \otimes+\frac{1}{2}=\left(\frac{1}{2} \otimes \otimes 1_{\mathscr{H}}\right. \\
& +\frac{1}{2} 1 \mathscr{H} \otimes+\frac{1}{2} \otimes \simeq+\frac{1}{2} \otimes 1_{\mathscr{H}}+\frac{1}{2} 1_{\mathscr{H}} \otimes
\end{aligned}
$$

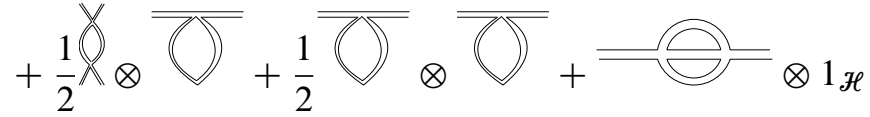

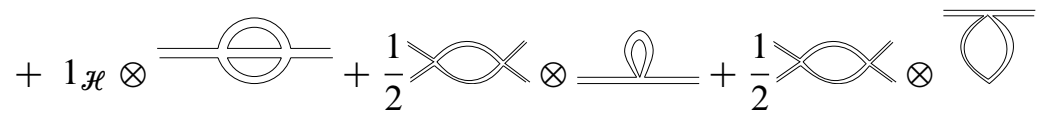




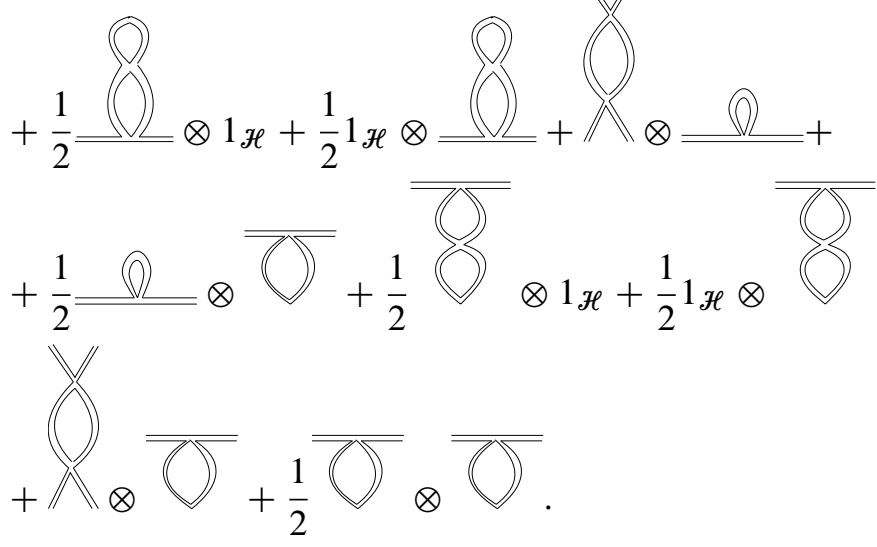

The right-hand side of Theorem 5.4 (ii) corresponding to the total contribution of the planar regular sector listed in (6.11) is worked out analogously, leading to

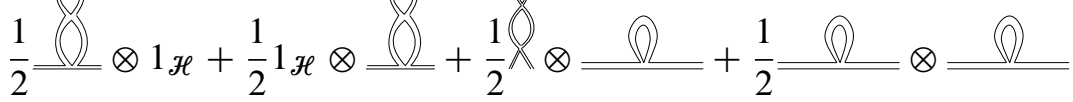

$$
\begin{aligned}
& +\frac{1}{2} \otimes 1_{\mathscr{H}}+\frac{1}{2} 1_{\mathscr{H}} \otimes+\frac{1}{2} \bar{\square} \otimes+\frac{1}{2} \otimes 1_{\mathscr{H}} \\
& +\frac{1}{2} 1 \mathscr{H}+\overline{(囚}+\frac{1}{2} \otimes 1_{\mathscr{H}}+\frac{1}{2} 1_{\mathscr{H}} \otimes
\end{aligned}
$$

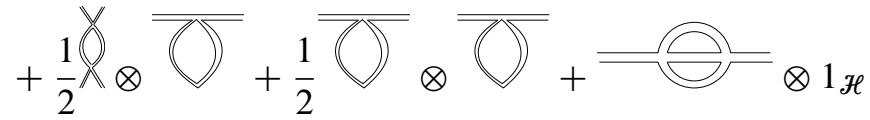

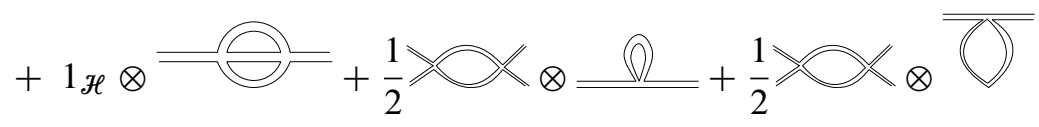

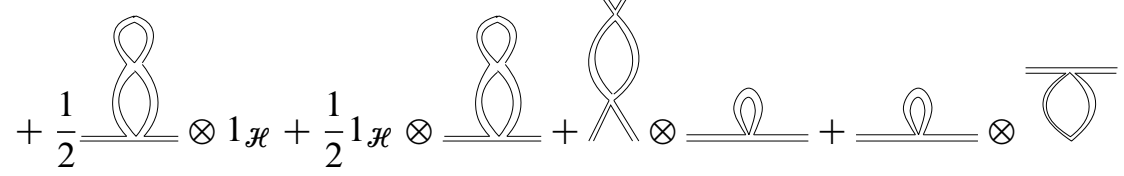

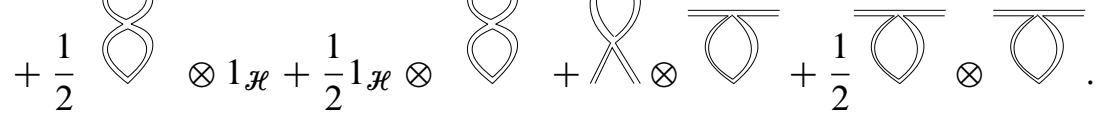


Comparing equations (6.14) and (6.15) above one is left on the right-hand side with

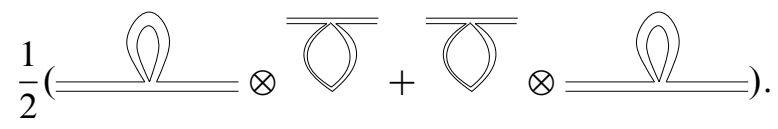

As above, the planar irregular sector $\tilde{c}_{1}$ saves the day. Indeed, when computing the left-hand side contribution associated to this new sector one has (using (6.8))

$$
\Delta B_{+}^{1,=}\left(\tilde{c}_{1}\right)=\frac{1}{2} \Delta(=+W
$$

Using again the definition (3.4) of the coproduct, (6.17) leads to

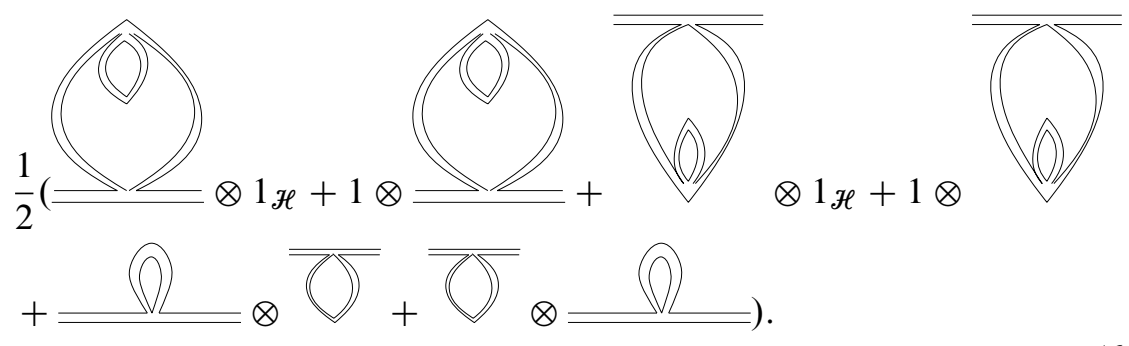

Let us now explicitly calculate the contribution of the new planar irregular sector $\tilde{c}_{1}$ to the right-hand side of Theorem 5.4 (ii). Using again (6.8) and discarding the planar irregular graphs from the final list, one gets

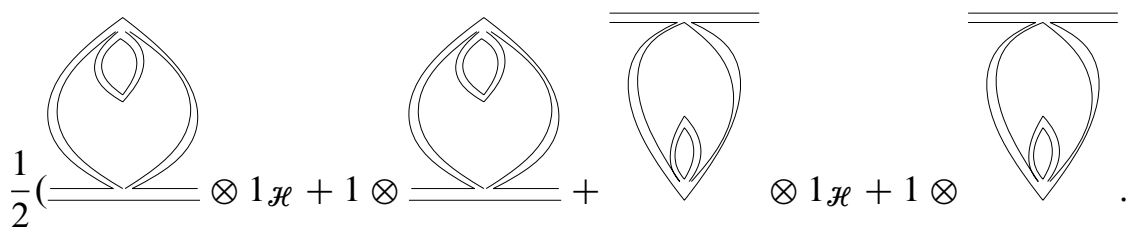

This cancels out with the left-hand side contribution of (6.18). One can thus see that the planar irregular sector has finally led to a total contribution

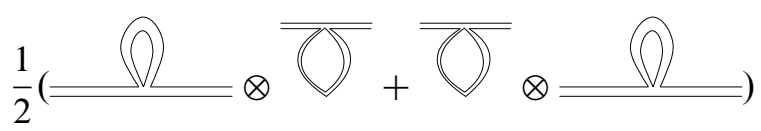

on the left-hand side of Theorem 5.4 (ii). This cancels out with the rest (6.16) of the planar regular sector. Let us remark that taking into consideration the planar irregular tadpole (6.10) does not change the situation since the insertion of this graph leads 
directly to non-planar graphs which are to be discarded. We have thus completely checked out Theorem 5.4 (ii) at the two-loop level, as announced.

Acknowledgment. A. T. acknowledges A. de Goursac, J. Magnen and V. Rivasseau for discussions. D. K. thanks K. Yeats for discussions. A. T. was partially suported by a Univ. Paris 13, Sorbonne Paris Cite "Bonus Qualité Recherche" grant and by grants PN 09370102 and CNCSIS “Tinere Echipe" 77/04.08.2010” D. K. was partially supported by NSF grant DMS-0603781.

\section{References}

[1] P. Aluffi and M. Marcolli, Feynman motives of banana graphs. Commun. Number Theory Phys. 3 (2009), 1-57. Zbl 1171.81384 MR 2504753

[2] J. Ben Geloun and A. Tanasa, One-loop $\beta$ functions of a translation-invariant renormalizable noncommutative scalar model. Lett. Math. Phys. 86 (2008), 19-32. Zbl 1175.81175 MR 2460724

[3] C. Bergbauer and D. Kreimer, Hopf algebras in renormalization theory: locality and Dyson-Schwinger equations from Hochschild cohomology. In Physics and number theory, IRMA Lect. Math. Theor. Phys. 10, Eur. Math. Soc., Zürich 2006, 133-164. Zbl 1141.81024 MR 2277758

[4] D. N. Blaschke, F. Gieres, E. Kronberger, M. Schweda, and M. Wohlgenannt, Translationinvariant models for non-commutative gauge fields. J. Phys. A 41 (2008), 252002. Zbl 1192.81326 MR 2454986; D. N. Blaschke, A. Rofner, M. Schweda and R. I. P. Sedmik, One-loop calculations for a translation invariant non-commutative gauge model. Eur. Phys. J. C Part. Fields 62 (2009), 433-443. Zbl 1188.81155 MR 2520536; D. N. Blaschke, A. Rofner, M. Schweda and R. I. P. Sedmik, Improved localization of a renormalizable non-commutative translation invariant $\mathrm{U}(1)$ gauge model. Europhys. Lett. 86 (2009), 51002.

[5] S. Bloch and D. Kreimer, Mixed Hodge structures and renormalization in physics. Commun. Number Theory Phys. 2 (2008), 637-718. Zbl 1214.81095 MR 2492196

[6] D. J. Broadhurst and D. Kreimer, Exact solutions of Dyson-Schwinger equations for iterated one-loop integrals and propagator-coupling duality. Nucl. Phys. B 600 (2001), 403-422. Zbl 1043.81049

[7] A. Connes, A. Connes, Noncommutative geometry. Academic Press, San Diego, CA, 1994.

Zbl 0818.46076 MR 1303779

[8] A. Connes and D. Kreimer, Renormalization in quantum field theory and the RiemannHilbert problem I: The Hopf algebra structure of graphs and the main theorem. Comm. Math. Phys. 210 (2000), 249-273. Zbl 1032.81026 MR 1748177

[9] A. Connes and M. Marcolli, Noncommutative geometry, quantum fields and motives. Amer. Math. Soc. Colloq. Publ. 55, Amer. Math. Soc., Providence, RI, 2008. Zbl 1209.58007 MR 2371808 
[10] A. de Goursac, A. Tanasa, and J.-C. Wallet, Vacuum configurations for renormalizable non-commutative scalar models. Eur. Phys. J. C Part. Fields 53 (2008), 459-466. Zbl 1189.81213 MR 2367062

[11] A. de Goursac, J.-C. Wallet, and R. Wulkenhaar, Noncommutative induced gauge theory. Eur. Phys. J. C Part. Fields 51 (2007), 977-987. Zbl 1189.81215 MR 2336186; $\mathrm{H}$. Grosse and M. Wohlgenannt, Induced gauge theory on a noncommutative space. Eur. Phys. J. C Part. Fields 52 (2007), 435-450. Zbl 1189.81217 MR 2346157; A. de Goursac, J.-C. Wallet and R. Wulkenhaar, On the vacuum states for non-commutative gauge theory. Eur. Phys. J. C Part. Fields 56 (2008), 293-304. Zbl 1189.81214 MR 2439583; D. N. Blaschke, H. Grosse and M. Schweda, Non-commutative U(1) gauge theory on $\mathbb{R}_{\Theta}^{4}$ with oscillator term and BRST symmetry. Europhys. Lett. 79 (2007), 61002. MR 2440992; D. N. Blaschke, H. Grosse, E. Kronberger, M. Schweda and M. Wohlgenannt, Loop calculations for the non-commutative $U_{\star}(1)$ gauge field model with oscillator term. Eur. Phys. J. C Part. Fields 67 (2010), 575-582.

[12] D. Dudal, J. A. Gracey, S. P. Sorella, N. Vandersickel and H. Verschelde, Refinement of the Gribov-Zwanziger approach in the Landau gauge: infrared propagators in harmony with the lattice results. Phys. Rev. D 78 (2008), 065047.

[13] H. Grosse and R. Wulkenhaar, Renormalisation of $\phi^{4}$-theory on noncommutative $\mathbb{R}^{4}$ in the matrix base. Comm. Math. Phys. 256 (2005), 305-374. Zbl 1075.82005 MR 2160797

[14] R. Gurau, J. Magnen, V. Rivasseau, and A. Tanasa, A translation-invariant renormalizable non-commutative scalar model. Comm. Math. Phys. 287 (2009), 275-290. Zbl 1170.81041 MR 2480749

[15] R. Gurau, J. Magnen, V. Rivasseau, and F. Vignes-Tourneret, Renormalization of noncommutative $\Phi_{4}^{4}$ field theory in $x$ space. Comm. Math. Phys. 267 (2006), 515-542. Zbl 1113.81101 MR 2249779

[16] R. Gurau, A. Malbouisson, V. Rivasseau, and A. Tanasa, Non-commutative complete Mellin representation for Feynman amplitudes. Lett. Math. Phys. 81 (2007), 161-175. Zbl 1161.81406 MR 2336230

[17] R. Gurau and V. Rivasseau, Parametric representation of noncommutative field theory. Comm. Math. Phys. 272 (2007), 811-835. Zbl 1156.81465 MR 2304476

[18] R. Gurău and A. Tanasă, Dimensional regularization and renormalization of noncommutative quantum field theory. Ann. Henri Poincaré 9 (2008), 655-683. Zbl 1147.81019 MR 2413199

[19] D. Kreimer, Anatomy of a gauge theory. Ann. Physics 321 (2006), 2757-2781. Zbl 1107.81038 MR 2274653

[20] D. Kreimer, Dyson-Schwinger equations: from Hopf algebras to number theory. In Universality and renormalization, Fields Inst. Commun. 50, Amer. Math. Soc., Providence, RI, 2007, 225-248. Zbl 05173770 MR 2310307

[21] D. Kreimer, The core Hopf algebra. In Quanta of maths, Clay Math. Proc. 11, Amer. Math. Soc., Providence, RI, 2010, 313-321. Zbl 1218.81085 MR 2732056

[22] D. Kreimer and W. D. van Suijlekom, Recursive relations in the core Hopf algebra. Nuclear Phys. B 820 (2009), 682-693. Zbl 1196.81199 MR 2554483

[23] D. Kreimer and K. Yeats, Recursion and growth estimates in renormalizable quantum field theory. Comm. Math. Phys. 279 (2008), 401-427. Zbl 1156.81033 MR 2383593 
[24] E. Langmann and R. J. Szabo, Duality in scalar field theory on noncommutative phase spaces. Phys. Lett. B 533 (2002), 168-177. Zbl 0994.81116 MR 1911944

[25] J. Magnen, V. Rivasseau and A. Tanasa, Commutative limit of a renormalizable noncommutative model. Europhys. Lett. 86 (2009), 11001.

[26] S. Minwalla, M. Van Raamsdonk, and N. Seiberg, Noncommutative perturbative dynamics. J. High Energy Phys. 2 (2000), 020. Zbl 0959.81108 MR 1748801

[27] V. Rivasseau, From perturbative to constructive renormalization. Princeton Ser. Phys., Princeton University Press, Princeton, NJ, 1991. MR 1174294

[28] V. Rivasseau and A. Tanasă, Parametric representation of "covariant" noncommutative QFT models. Comm. Math. Phys. 279 (2008), 355-379. Zbl 1158.81026 MR 2383591

[29] A. Tanasa, Feynman amplitudes in renormalizable non-commutative quantum field theory. Preprint 2007. arXiv:0711.3355

[30] A. Tanasa, Overview of the parametric representation of renormalizable non-commutative field theory. J. Phys. Conf. Ser. 103 (2008), 012012.

[31] A. Tanasa, Parametric representation of a translation-invariant renormalizable noncommutative model. J. Phys. A 42 (2009), 365208. Zbl 1178.81262 MR 2534516; T. Krajewski, V. Rivasseau, A. Tanasă, and Z. Wang, Topological graph polynomials and quantum field theory. Part I: Heat kernel theories. J. Noncommut. Geom. 4 (2010), 29-82. Zbl 1186.81095 MR 2575389

[32] A. Tanasa, Scalar and gauge translation-invariant noncommutative models. Romanian J. Phys. 53 (2008), 1207-1212. Zbl 1187.81200 MR 2470786

[33] A. Tanasă and F. Vignes-Tourneret, Hopf algebra of non-commutative field theory. $J$. Noncommut. Geom. 2 (2008), 125-139. Zbl 1181.81101 MR 2366125

[34] F. Vignes-Tourneret, Renormalization of the orientable non-commutative Gross-Neveu model. Ann. Henri Poincaré 8 (2007), 427-474. Zbl 1133.81058 MR 2329358

Received July 13, 2009; revised January 13, 2011

A. Tanasă, Laboratoire d'Informatique de Paris-Nord, UMR CNRS 7030, Institut Galilée - Université Paris-Nord, 99, avenue Jean-Baptiste Clément, 93430 Villetaneuse, France; and Horia Hulubei National Institute for Physics and Nuclear Engineering, P.O.B. MG-6, 077125 Magurele, Romania

E-mail: Adrian.Tanasa@lipn.univ-paris13.fr

D. Kreimer, Humboldt Universität zu Berlin, Institut für Physik und Institut für Mathematik, Newton Strasse 15, 12489 Berlin, Germany

E-mail: kreimer@mathematik.hu-berlin.de 